\title{
Analysis and decomposition of condensed tannins in tree leaves
}

The decomposition of leaf litter is controlled by several factors. One factor that may play an important role is the content of condensed tannins (proanthocyanidins). Here we designed a combined method to isolate proanthocyanidins from leaf extracts, to convert them to anthocyanidins, and to quantify individual anthocyanidins exactly with a new simple, but sensitive highperformance liquid chromatography method. We used this method to show composition of proanthocyanidins and to monitor degradation of proanthocyanidins and individual constituents in leaf litter in an aquatic environment over time. Despite the rapid decrease in the initial concentrations, a fraction of the proanthocyanidins remained detectable for several weeks.

Keywords Polyphenolic compounds, Proanthocyanidins, Anthocyanidins, Leaf litter decomposition, Tannins, Degradation of condensed tannins

\section{Introduction}

In the past decades, many studies have shown that tannins (polyphenolic compounds) are involved in defence mechanisms of plants against attack by bacteria, fungi, and herbivores. Little is known about the mechanisms of action of the polyphenolic compounds (Zucker 1983, Scalbert 1991) even though modern analytical methods have improved the analysis of these complex structures (Mole and Watermann 1987, Schofield et al. 2001). Proposals on the mechanism of action include tannins forming stable complexes with plant proteins to make the tissue unattractive and difficult to digest (Schofield et al. 2001), and tannins acting like a toxin through highly specific reactions with digestive enzymes or directly at the cell membranes (Zucker 1983) or through depletion of essential iron by complexation (Mila et al. 1996).

Such mechanisms are not only important as a defence in living plants, but may also control terrestrial and aquatic decomposition processes, provided that the effective substances remain in the dead tissues. Indeed, leaves with high initial contents of polyphenolic compounds, especially of condensed tannins (proanthocyanidins), seem to decompose slowly in both terrestrial (Valachovic et al. 2004) and aquatic ecosystems (Wantzen et al. 2002). However, only strong relationships but not significant correlations between the initial content of total polyphenolic compounds or tannins and the velocity of litter decomposition have been demonstrated in most studies, which indicates that multiple causes of delayed decomposition are likely.

The analytical methods for the quantitative determination of polyphenolic compounds have been reviewed (Mole and Waterman 1987, Hagermann 1998, Schofield et al. 2001). Simple photometric methods for the determination of total phenols have been preferred over substance-specific methods, e.g., for proanthocyanidins, in ecological studies on leaf litter decomposition. Such general methods, however, have considerable drawbacks (Schofield et al. 2001). Both the isolation and the precise analysis of proanthocyanidins are very complex and timeconsuming. Hence, nearly all of the studies report only photometrically determined initial contents of total phenols. Initial values of proanthocyanidins or other fractions of polyphenols have been reported in some studies, but proanthocyanidin degradation over time has not been analysed.

Proanthocyanidins may play an important role in aquatic leaf litter decomposition, as they may deter invertebrate shredders (Wantzen et al. 2002). Moreover, they may retard the "conditioning" of leaves by fungi and bacteria. To test this hypothesis, analytical data of the long-term decomposition of leaf litter, e.g., analysis of the change in the content of proanthocyanidins in decomposing plant material, are needed. Here we describe a method to extract and precisely determine proanthocyanidins from decomposing tree leaves. The method is used to follow the decomposition over time of the leaves of three tree species common to three tropical aquatic environments.

Karen Haase, Karl Matthias Wantzen

Max-Planck Institute for Limnology, Tropical Ecology Working Group,

P.O. Box 165, D-24306 Plön, Germany

haase@mpil-ploen.mpg.de 


\section{Experimental}

Plant material

Leaves of three common tree species were used for the decomposition experiments: Symmeria paniculata (Polygonaceae) from an Amazonian white-water floodplain forest in Colombia, Nectandra amazonum (Lauraceae) from a floodplain in Argentina, and Macaranga pruinosa (Euphorbiaceae) from a peat swamp forest in Malaysia.

\section{Decomposition experiment}

Litterbags with $5 \mathrm{~g}$ of fresh air-dried leaves were exposed in a moderately flowing stretch of streams at the tropical sites. A randomly selected set of three litterbag samples per species was retrieved after $7,14,28$, and 56 days at all sites, and additionally after 128 days in Argentina and after 6 and 12 months in Malaysia. For the chemical analyses, sampled leaves were cleaned, air-dried, weighed, and stored under dry conditions.

Extraction

Air-dried leaves were cut into small pieces $(5-8 \mathrm{~mm})$ or ground $(<200 \mu \mathrm{m})$ and then dried in a desiccator to a constant weight. Leaf material (600-800 mg) was extracted with $25 \mathrm{ml}(28 \mathrm{mg} / \mathrm{ml}$ tissueto-solvent ratio) or $50 \mathrm{ml}(14 \mathrm{mg} / \mathrm{ml})$ of $60 \%$ aqueous acetone (60/40, by vol) in an ice bath for 15 min with an Ultra-Turrax T 25 homogenizer (Janke \& Kunkel, Germany). Samples were centrifuged for $25 \mathrm{~min}(10000 \mathrm{rpm}$, rotor radius $15.5 \mathrm{~cm})$, the supernatant was removed, and the extraction was repeated. Supernatants were combined and then distilled under reduced pressure to give a finite volume of aqueous raw extract.

Isolation and quantitative determination of proanthocyanidins

The water-soluble oligomeric fractions of the proanthocyanidins were separated following a slight modification of the procedure proposed by Sarni-Manchado et al. (1999). Raw extract $(10 \mathrm{ml})$ filtered through a $0.4 \mu \mathrm{m}$ filter was applied to a column (length $15 \mathrm{~cm}$, diameter $3 \mathrm{~cm}$, gel volume $110 \mathrm{ml}$ ) of Toyopearl resin HW50F (Tosoh Bioscience, Stuttgart, Germany). The column was then first washed with $200 \mathrm{ml}$ water $(3 \mathrm{ml} / \mathrm{min})$, and then with $300 \mathrm{ml}$ ethanol/water/trifluoroacetic acid (550/440/0.5, by vol). Unwanted fractions, e.g. simple phenols and monomeric polyphenolic compounds, such as phenolic acids, anthocyanins, flavonols and their glycosides were removed by this procedure. The oligomeric proanthocyanidin fraction was recovered with $200 \mathrm{ml}$ of $60 \%$ acetone. Acetone was removed by distillation under reduced pressure, and the aqueous oligomer solution was frozen and freezedried for about $50 \mathrm{~h}$. The dry samples were dissolved in 10-30 ml of ethanol, and the oligomeric chains were cleaved oxidatively (1 h) with $\mathrm{HCl}$ and ferric ions following the procedure of Porter et al. (1986). The samples were then analysed by high performance liquid chromatography (Thermo gradient pump P 2000, UV/VIS detector UV 6000, Thermo Electron Corporation, USA). A Beertype relationship between the absorbance of the released coloured anthocyanidins (Fig. 1, molecular structure) and the concentration of oligomers in the ethanolic solutions is only applicable in the lower oligomer concentration range $(<150-200 \mathrm{mAU})$. The released coloured anthocyanidins were analysed using a reversedphase Nucleosil $100-5 \mathrm{C}_{18}$ column $(250 \times 4 \mathrm{~mm}$, Macherey-Nagel, Düren, Germany) at different wavelengths according to the maximum wavelengths of the commercially available anthocyanidin standards pelargonidin chloride $(512 \mathrm{~nm})$, cyanidin chloride $(524 \mathrm{~nm})$, peonidin chloride $(524 \mathrm{~nm})$, and delphinidin chloride (532 nm; Extrasynthese, Genay, France). The mobile phase consisted of (A) water/formic acid (980/20, v/v) and (B) acetonitrile/water/formic acid (800/180/20, by vol). Samples were eluted at a flow rate of $1.1 \mathrm{ml} / \mathrm{min}$ with a linear gradient: $0-4 \mathrm{~min}$ $100 \% \mathrm{~A} ; 5 \min 85 \% \mathrm{~A} / 15 \% \mathrm{~B} ; 20 \min 35 \% \mathrm{~A} / 65 \% \mathrm{~B} ; 30 \mathrm{~min}$ $35 \% \quad \mathrm{~A} / 65 \% \mathrm{~B} ; 35 \mathrm{~min} 100 \%$ A (Rainer Suetfeld, personal communication).

Data analysis

Exponential functions corresponding to proanthocyanidin degradation and elution with negative exponential coefficients (k') were calculated using a non-linear fitting procedure (SigmaPlot statistics). Three to four parallel samples were analysed on five sampling dates in Colombia and six sampling dates in Argentina and Malaysia. The coefficient k' is a measure of the velocity of proanthocyanidin loss in analogy to the leaf litter decomposition coefficient $\mathrm{k}$, which is usually used to quantify the leaf litter decomposition process (see, e.g. Valachovic et al. 2004).

\section{Results and discussion}

Quantitative determination of proanthocyanidins in leaf litter

We set out to determine the optimal conditions for proanthocyanidin extraction and an optimal method for the determination of proanthocyanidins in a leaf raw extract using the procedure for the isolation of oligomeric proanthocyanidins (SarniManchado 1999) and the method for the oxidative cleavage of the oligomeric chains (Porter et al. 1986) as a basis, combined with HPLC analysis of the individual released anthocyanidins.

The traces of the reversed-phase high performance liquid chromatography obtained were specific for the tree species. The traces showed between 3 (decomposed leaves) and 15 (initial leaves) well-separated and symmetrical peaks (Fig. 1).

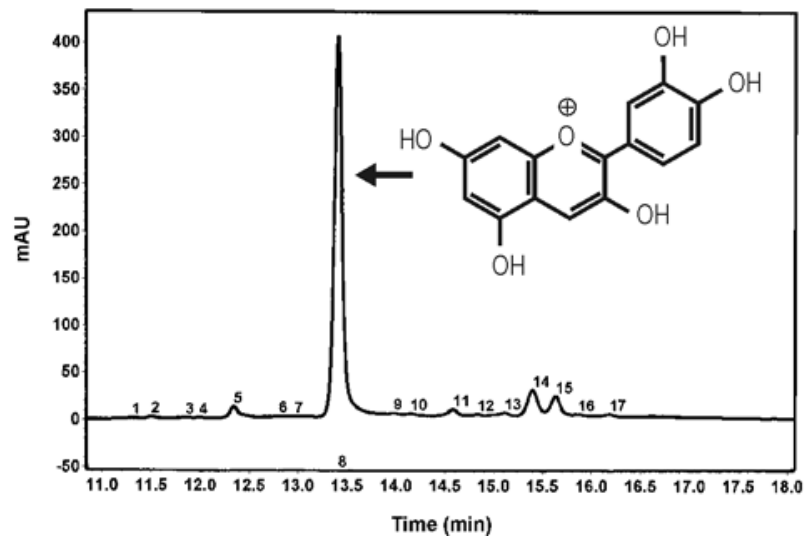

Fig. 1 Trace $(524 \mathrm{~nm})$ of anthocyanidins separated by reversedphase chromatography. The oligomeric fraction of the proanthocyanidins of Macaranga pruinosa (initial leaves) was first cleaved oxidatively. 5: delphinidin, 8: cyanidin, 11: pelargonidin 
Four peaks were assigned to the commercially available anthocyanidins delphinidin, cyanidin, pelargonidin and peonidin and were quantified separately. Cyanidin was the main anthocyanidin in all leaf extracts analysed, followed by pelargonidin and/or delphinidin and in some extracts peonidin. The other, unidentified peaks in all samples were of negligible importance for the quantitative analysis. In this study the sum of the quantified anthocyanidins represented the total extractable proanthocyanidins. The concentrations of proanthocyanidin determined are probably slightly lower than the actual concentration for three reasons: (1) we cannot exclude that a fraction of non-cleavable proanthocyanidins was not registered, e.g., 5-deoxy-proanthocyanidins are not readily cleaved by this procedure (Schofield 2001); (2) in most cases, some unidentified monomers in very low amounts (for which no standards are commercially available) were not quantified; and (3) the oligomer concentrations determined, which are based on monomeric standards, underestimate proanthocyanidins because the terminal units of the oligomers do not give rise to a coloured anthocyanidin monomer; this problem minimizes with the chain length of the oligomers.

We tested whether grinding the leaves of the three tree species increased the amount of proanthocyanidins extracted. Unground leaves of Nectandra microcarpa contained $1141 \mu \mathrm{g}$ proanthocyanidins per $\mathrm{g}$ leaf dry weight $(\mathrm{SD}=91 \mu \mathrm{g} / \mathrm{g})$. Grinding of the leaf material lowered the variability and increased the yield of total extractable proanthocyanidins 1.8-fold. Similar results (2fold increase in the yield) were obtained with leaves of Macaranga pruinosa, but the yields obtained from ground and unground leaves of Symmeria paniculata did not differ. Using ground leaves, the proanthocyanidin content (mean values of three parallel samples) at $\mathrm{t}=0$ were: Nectandra microcarpa, $2050 \mu \mathrm{g} / \mathrm{g}$, Symmeria paniculata, $54.6 \mathrm{mg} / \mathrm{g}$, and Macaranga pruinosa, $19.04 \mathrm{mg} / \mathrm{g}$. The sums of the total proanthocyanidin obtained are in the range of previously reported values determined using the butanol- $\mathrm{HCl}$ colorimetric reaction (Valachovic et al. 2004).

Other parameters of the extraction procedure were less critical. Lowering of the tissue-to-solvent ratio from $28 \mathrm{mg} / \mathrm{ml}$ to $14 \mathrm{mg} / \mathrm{ml}$ did not increase the extraction yield from leaves of the three tree species tested. However, we suggest that optimal conditions for maximal extraction and cleavage yields be experimentally determined in studies of other species because the variation in the fixation and composition of the proanthocyanidins may alter the readiness of extraction and of carbon-carbon bond cleavage.

Our combined method provides a means to analyse with high accuracy only that fraction of tannins with the highest probability of having a steady influence on decomposition, i.e. only proanthocyanidins. In contrast to the usually used photometric methods, which often overestimate the fraction of condensed tannins (Mole and Waterman 1987, Schofield 2001), our method detects only individual, coloured anthocyanidins released from the oligomeric proanthocyanidins, each measured at the optimal wavelength. Monomeric flavanoids in the extract, which possibly have no physiological effect (Scalbert 1991), are removed during column chromatography. The determination of individual anthocyanidins additionally offers a means of relating the specific proanthocyanidin composition to the characteristics of the decomposition process.

Degradation of proanthocyanidins during decomposition
We used our method to analyse the proanthocyanidins in the leaves of the three tree species during decomposition over time. The leaves of the selected species had different initial $(\mathrm{t}=0)$ proanthocyanidin contents, and the proanthocyanidin contents decreased rapidly over time (Fig. 2, symbols).
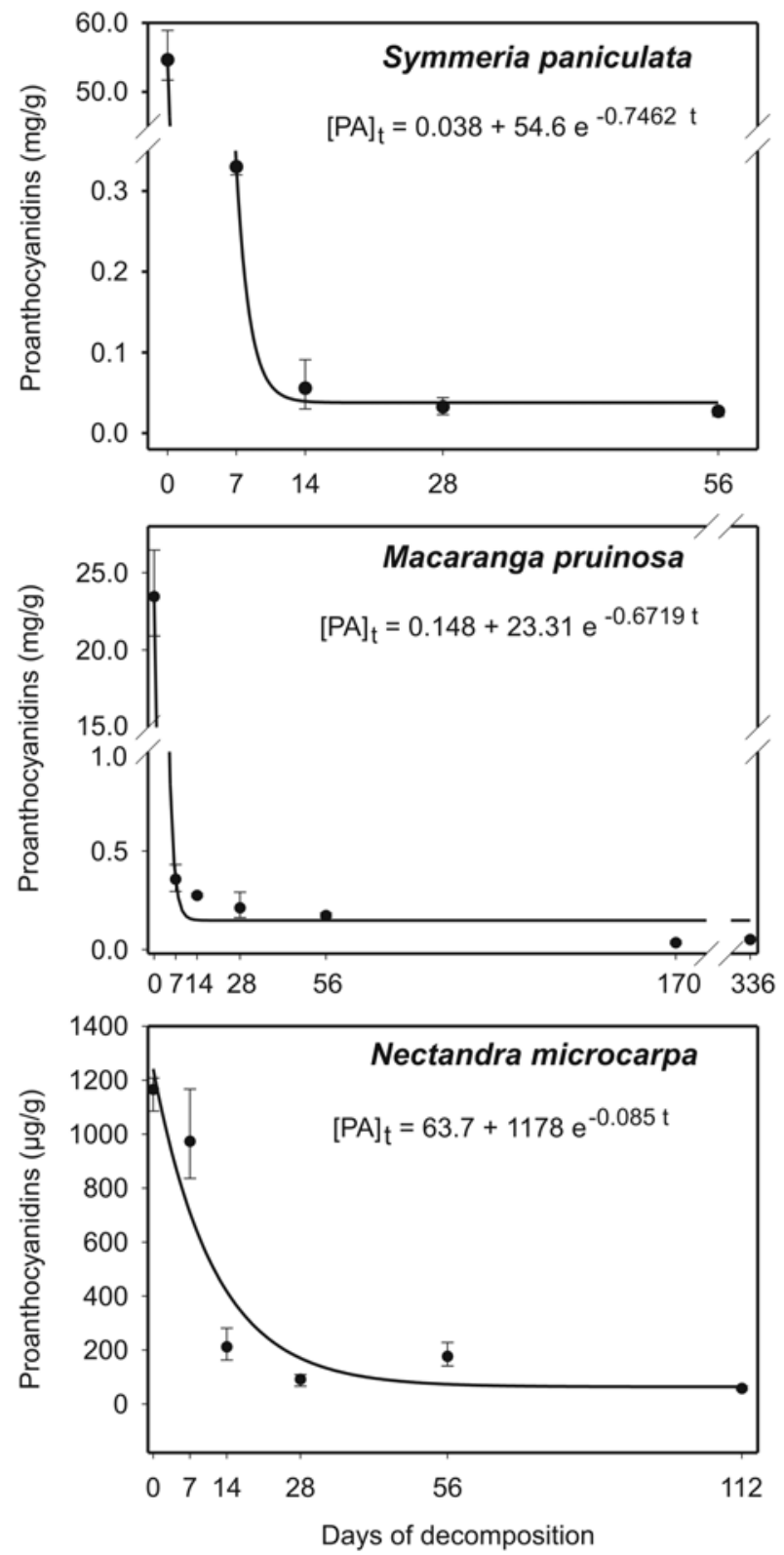

Fig. 2 Degradation of extractable proanthocyanidins in leaves of the three tree species indicated during decomposition in a stream. The data points represent the mean values of three parallel samples. The curves are the calculated exponential degradation functions

After a rapid decrease, the proanthocyanidin contents approached a remaining value asymptotically, which suggested a negative exponential time course corresponding to the simple function $[\mathrm{PA}]_{\mathrm{t}}=[\mathrm{PA}]_{\mathrm{rest}}+[\mathrm{PA}]_{0} \mathrm{e}^{-\mathrm{k}^{\prime} \mathrm{t}}$, where $[\mathrm{PA}]_{\mathrm{t}}$ is the proanthocyanidin content at a certain time point, $[\mathrm{PA}]_{0}$ is the initial proanthocyanidin content, $[\mathrm{PA}]_{\text {rest }}$ is the remaining proanthocyanidin content, $\mathrm{t}$ is the time, and $\mathrm{k}$ ' is the proanthocyanidin degradation coefficient representing the 
proanthocyanidin degradation velocity. Adjusting this function to the analysed proanthocyanidin contents by non-linear fitting results in exponential functions that match the experimental data well (Fig. 2, curves).

The calculated proanthocyanidin degradation functions vary considerably in their parameters between the species, which suggested differences in the composition of the oligomeric chains and in their fixation in the tissue of the dead leaves. The proanthocyanidin contents of Symmeria paniculata und Macaranga pruinosa were very high but decreased relatively fast, whereas the content of Nectandra microcarpa was only relatively moderate and the degradation was very slow. In all three species, considerable amounts of proanthocyanidins remained in the leaf tissue in water for at least 56 days, which suggested that the condensed tannins were stabilized by further polymerisation and/or formation of stable complexes to proteins, polysaccharides, and other macromolecules in the dead leaf tissue. Highly polymerised condensed tannins with chain lengths greater than 10-12, which might be produced during decomposition by secondary changes in short-chain tannins, cannot be extracted under the conditions used. Thus, the remaining proanthocyanidin content is probably higher than calculated.

Of the individual anthocyanidins detected, delphinidin was eluted very fast (Fig. 3). After seven days of decomposition in the stream, only traces could be detected in leaves of all species.

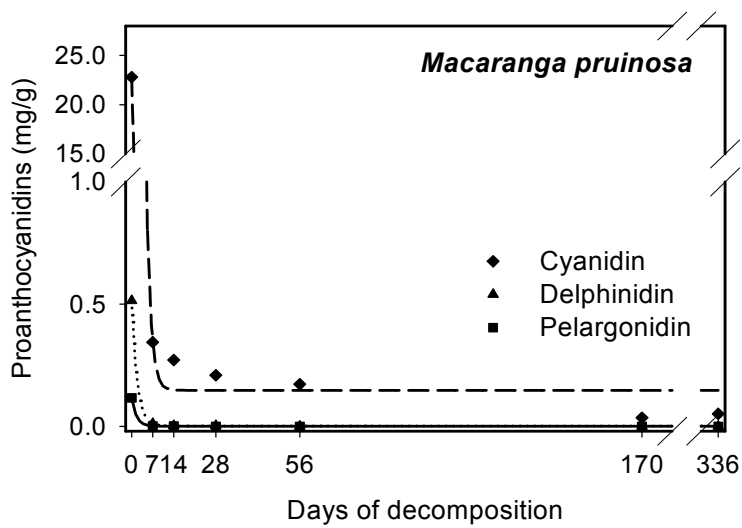

Fig. 3 Mean values of three parallel samples and the calculated exponential degradation functions of individual anthocyanidins in leaves of Macaranga pruinosa

Thus, proanthocyanidins with high amounts of delphinidin, which have been considered by some authors as the most effective components in forming complexes for instance with proteins because of their high number of hydroxyl groups (Scalbert 1991, Schofield 2001), cannot play an appreciable role in the control of the aquatic decomposition process since their enhanced water solubility (three hydroxyl groups) resulted in a rapid loss in the decomposing leaves. The other, not yet identified anthocyanidins were also degraded very rapidly. After a long decomposition period, almost exclusively cyanidin was detected in the leaf extracts, which points toward procyanidins having particular importance during the decomposition processes.

In ecological studies investigating the relationship between condensed tannins and the velocity of leaf litter decomposition, the mathematical parameters of the proanthocyanidin degradation functions could be used in combination with the initial proanthocyanidin contents for correlation analyses.

\section{Conclusion}

The presented method is suitable for reliable analysis of proanthocyanidins. The separation of fragments by a simple highperformance liquid chromatography method with high sensitivity of the visual detection is advantageous versus usually used overall photometric methods. Monitoring of proanthocyanidins in decomposing leaves with this method provides the content and the composition of this fraction of tannins over time. Those analytical data allow for testing the influence of these compounds on aquatic leaf litter decomposition. The remaining procyanidins in the leaves of many species after weeks of decomposition suggest a potential for a long-lasting effect on aquatic decomposition.

\section{Acknowledgements}

This study is a result of WW-DECOEX, a worldwide comparison experiment on aquatic leaf litter decomposition. We thank Guillermo Rueda-Delgado (UTADEO, Colombia), Mercedes Marchese (INALI, Santa Fé, Argentina), and Catherine Yule (Monash University, Kuala Lumpur, Malaysia) for contributing leaf samples from their research sites. This study received financial support by the Deutsche Forschungsgemeinschaft (DFG WA 1612/2-1). Publication No. 3 of the WW-DECOEX project. We dedicate this paper to the memory of the late Rainer Suetfeld.

\section{References}

Hagerman AE (1998) Tannin analysis. www.users.muohio.edu/hagermae/tannin.pdf

Mila I, Scalbert A, Expert D (1996) Iron withholding by plant polyphenols and resistance to pathogens and rots. Phytochemistry 42 (6): 1551-1555

Mole S, Watermann PG (1987) A critical analysis of techniques for measuring tannins in ecological studies. Oecologia 72: 137-147 Porter LJ, Hrstich LN, Chan BG (1986) The conversion of procyanidins and prodelphinidins to cyanidin and delphinidin. Phytochemistry 25 (1): 223-230

Sarni-Manchado P, Deleris A, Avallone S, Cheynier V, Moutounet M (1999) Analysis and characterization of wine condensed tannins precipitated by proteins used as fining agent in enology. Am J Enol Vitic 50 (1): 81-86

Scalbert A (1991) Antimicrobial properties of tannins. Phytochemistry 30 (12): 3875-3883

Schofield P, Mbugua DM, Pell AN (2001) Analysis of condensed tannins: a review. Animal Feed Science and Technology 91: 21-40 Valachovic YS, Caldwell BA, Cromack K, Griffiths RP (2004) Leaf litter chemistry controls on decomposition of Pacific Northwest trees and woody shrubs. Can J For Res 34: 2131-2147

Wantzen KM, Wagner R, Suetfeld R, Junk WJ (2002) How do plant-herbivore interactions of trees influence coarse detritus processing by shredders in aquatic ecosystems of different latitudes? Verh Int Verein Limnol 28: 815-821

Zucker WV (1983) Tannins: Does structure determine function? An ecological perspective. The American Naturalist 121 (3): 335365 